\title{
Specific and Differential Activation of Mitogen-Activated Protein Kinase Cascades by Unfamiliar Taste in the Insular Cortex of the Behaving Rat
}

\author{
Diego E. Berman, ${ }^{1}$ Shoshi Hazvi, ${ }^{1}$ Kobi Rosenblum, ${ }^{1}$ Rony Seger, ${ }^{2}$ and Yadin Dudai ${ }^{1}$ \\ Departments of ${ }^{1}$ Neurobiology and ${ }^{2}$ Biological Regulation, The Weizmann Institute of Science, Rehovot 76100, Israel
}

\begin{abstract}
Rats were given to drink an unfamiliar taste solution under conditions that result in long-term memory of that taste. The insular cortex, which contains the taste cortex, was then removed and assayed for activation of mitogen-activated protein kinase (MAPK) cascades by using antibodies to the activated forms of various MAPKs. Extracellular responsive kinase 1-2 (ERK1-2) in the cortical homogenate was significantly activated within $<30$ min of drinking the taste solution, without alteration in the total level of the ERK1-2 proteins. The activity subsided to basal levels within $<60$ min. In contrast, ERK1-2 was not activated when the taste was made familiar. The effect of the unfamiliar taste was specific to the insular cortex. Jun $\mathrm{N}$-terminal kinase 1-2 (JNK1-2) was activated by drinking the taste but with a delayed time course, whereas the activity of Akt kinase and p38MAPK remained unchanged. Elk-1, a member of
\end{abstract}

the ternary complex factor and an ERK/JNK downstream substrate, was activated with a time course similar to that of ERK1-2. Microinjection of a reversible inhibitor of MAPK/ERK kinase into the insular cortex shortly before exposure to the novel taste in a conditioned taste aversion training paradigm attenuated long-term taste aversion memory without significantly affecting short-term memory or the sensory, motor, and motivational faculties required to express long-term taste aversion memory. It was concluded that ERK and JNK are specifically and differentially activated in the insular cortex after exposure to a novel taste, and that this activation is required for consolidation of long-term taste memory.

Key words: taste; incidental learning; conditioned taste aversion; long-term memory; insular cortex; mitogen-activated protein kinase; novelty
Taste learning in the rat is rapid and robust. This might be attributable to the fact that rodents are immersed in a world dominated by chemical information (Revusky and Garcia, 1970; Bures et al., 1988). Behavioral paradigms that tax taste memory in the rat, when combined with cellular and molecular methods, offer therefore significant advantages in the analysis of learning and memory (Rosenblum et al., 1993, 1997; Yamamoto et al., 1994; Schafe et al., 1995; Lamprecht and Dudai, 1997). When a rat encounters an unfamiliar taste, information flows to the central taste area in the insular cortex (Braun et al., 1982; Finger, 1987; Hettinger and Frank, 1992) and triggers there molecular changes that subserve the encoding of taste memory. These changes involve tyrosine phosphorylation of the $2 \mathrm{~B}$ subunit of the NMDA receptor (Rosenblum et al., 1997), activation of protein kinase C (Bahar and Dudai, 1997; Yasoshima and Yamamoto, 1997), cholinergic modulation (Naor and Dudai, 1996), and protein synthesis (Rosenblum et al., 1993). Specifically, the ability of protein synthesis inhibitors to block the formation of taste memory in cortex in the first few hours after sampling a novel taste (ibid.) fits into a conceptual framework that depicts consolidation of long-term memory as dependent on modulation of gene expression (Goelet et al., 1986) and synaptic resculpture (Bailey and Kandel, 1993).

The possibility that synaptic remodeling subserves taste mem-

\footnotetext{
Received June 3, 1998; revised Sept. 3, 1998; accepted Sept. 10, 1998.

This work was supported by grants from the Carl Dominic Center for Brain Research, the Abramson Fund, and the Reich Fund. We thank Raphael Lamprecht and Sima Lev for helpful comments on this manuscript.

Correspondence should be addressed to Dr. Yadin Dudai, Department of Neurobiology, The Weizmann Institute of Science, Rehovot 76100, Israel.

Copyright (C) 1998 Society for Neuroscience $\quad 0270-6474 / 98 / 1810037-08 \$ 05.00 / 0$
}

ory in the insular cortex has led us to search for molecular mechanisms that might link the acquisition of taste information to lasting changes in the cortical circuits, which are presumed to retain the representation of taste over time. In this context, mitogen-activated protein kinase (MAPK) cascades are attractive candidates. MAPKs are families of Ser/Thr protein kinases, which take part in transmission, classification, and amplification of information embedded in extracellular signals from the membrane via the cytoplasm to the nucleus (Cano and Mahadevan, 1995; Seger and Krebs, 1995; Sugden and Clerk, 1997). In many types of tissues, MAPKs contribute to proliferation, differentiation, and development (ibid.). In neural systems, MAPKs were implicated in plasticity and learning. In long-term facilitation in Aplysia, apMAPK [homolog of the human extracellular responsive kinase 2 (ERK2)] was shown to be activated by serotonin (Michael et al., 1998) and translocated into the neuronal nucleus (Martin et al., 1997). Furthermore, in the same system a mutation in the phosphorylation sites of apMAPK blocked learning-related internalization of apCAM (Bailey et al., 1997). ERK activation was also reported to play a role in conditioning in Hermissenda (Crow et al., 1998) and in hippocampal long-term potentiation (LTP) (English and Sweatt, 1996).

We have therefore investigated whether taste-learning experience modulates the activity of MAPKs in the insular cortex. Here, we report that activation of MAPKs in the insular cortex of the behaving rat is correlated with, and obligatory for, normal taste learning in both incidental and associative situations. The effect is specific to an unfamiliar (as opposed to familiar) taste and to the insular cortex and is differential; namely, only ERK1-2 and Jun N-terminal kinase 1-2 (JNK1-2), but not Akt kinase and p38MAPK, are activated. 


\section{MATERIALS AND METHODS}

Animals. Male Wistar rats ( $\sim 60$-d-old, 200-250 gm) were used. They were caged individually at $22 \pm 2^{\circ} \mathrm{C}$ in a $12 \mathrm{hr}$ light/dark cycle.

Reagents. Monoclonal anti-diphospho ERK (dpERK) (Thr183/Tyr185, MAPK-YT) and anti-ERK antibodies were from Sigma (St. Louis, MO). Polyclonal anti-phospho Akt (pAkt) (Ser473), anti-Akt, anti-phospho Elk-1 (pElk-1) (Ser383), anti-Elk-1, anti-diphospho JNK (dpJNK) (Thr183/Tyr185), anti-JNK, anti-diphospho p38MAPK (dp-p38MAPK) (Thr180/Tyr182), and anti-p38MAPK were from New England Biolabs (Beverly, MA). Horseradish peroxidase (HRP)-linked protein A and the enhanced chemiluminescense (ECL) kit were from Amersham (Buckingshamshire, UK). Goat anti-mouse ( $\mathrm{IgG}$ ) peroxidase conjugate was from Sigma. PD098059 was from Calbiochem (La Jolla, CA). All other chemicals were of analytical grade or the highest grade available.

Behavioral procedures. Throughout the study, we used two types of taste learning situations: incidental (Hebb, 1949) and associative (Garcia et al., 1955). Incidental learning was used for most of the molecular analysis of the effect of taste experience on MAPKs in insular cortex. The rats were exposed once for $10 \mathrm{~min}$ to an unfamiliar taste (Rosenblum et al., 1993), followed by decapitation at the indicated times and tissue processing as described below. The behavioral consequences of such exposure, i.e., the formation of memory for the novel taste, was determined in other groups of rats by using the associative conditioned taste aversion paradigm (CTA) (Revusky and Garcia, 1970; Bures et al., 1988), either alone or in combination with latent inhibition (see below) (Lubow, 1989). In CTA, organisms learn to avoid a novel taste if its ingestion is followed by transient poisoning. We used the procedure detailed in Rosenblum et al. (1993, 1997). Unless indicated otherwise, saccharin $(0.1 \% \mathrm{w} / \mathrm{v}$, sodium salt) was used as the unfamiliar taste in training, i.e., the conditioned stimulus (CS), and injection of $\mathrm{LiCl}(0.15 \mathrm{M}, 2 \%$ body weight, i.p.) as the malaise-inducing agent (the unconditioned stimulus, US). Rats were pretrained for $3 \mathrm{~d}$ to get their daily water ration once a day for $10 \mathrm{~min}$ from two pipettes containing $10 \mathrm{ml}$ of water. On the conditioning day, they were allowed to drink the saccharin solution instead of water from similar pipettes for $10 \mathrm{~min}$, and $40 \mathrm{~min}$ after the offset of the drinking period they were injected intraperitoneally with LiCl. Under these conditions, $3 \mathrm{~d}$ after training the conditioned rats preferred water to saccharin at a ratio of approximately 9:1 in a multiple choice test situation (three pipettes with $5 \mathrm{ml}$ of saccharin each, three pipettes with $5 \mathrm{ml}$ of water each), whereas nonconditioned rats preferred saccharin to water. The behavioral data are presented below in terms of aversion index (AI), defined as $\{[$ milliliters of water/(milliliters of water + milliliters of saccharin)] x 100\} consumed in the test; 50 is chance level, and the higher the AI, the more the rats prefer water to the conditioned taste.

In some experiments, a latent inhibition procedure (Lubow, 1989) was used to measure familiarity. The combination of CTA and latent inhibition takes advantage of the fact that CTA is much more effective if the $\mathrm{CS}$ is unfamiliar. In latent inhibition, preexposure to a sensory stimulus attenuates the effectiveness of that same stimulus as a CS in subsequent learning. Thus, exposure of rats to an unfamiliar taste several days before CTA training significantly reduces the acquired aversion to that same taste (Rosenblum et al., 1993, 1997). In latent inhibition experiments, the rats were exposed for $10 \mathrm{~min}$ to either saccharin or water (control), in two pipettes of $10 \mathrm{ml}$ each, $3 \mathrm{~d}$ before the second exposure to saccharin, as the CS in CTA training.

Surgery and microinjection. Rats were anesthetized with $5.6 \mathrm{ml} / \mathrm{kg}$ Equithesin $\left(2.12 \% \mathrm{w} / \mathrm{v} \mathrm{MgSO}_{4}, 10 \% \mathrm{v} / \mathrm{v}\right.$ ethanol, $39.1 \% \mathrm{v} / \mathrm{v}$ propylene glycol, $0.98 \% \mathrm{w} / \mathrm{v}$ sodium pentobarbitone, $4.2 \% \mathrm{w} / \mathrm{v}$ chloral hydrate), restrained in a stereotaxis apparatus (Kopf), and implanted bilaterally with a stainless steel guide cannula (23 gauge) aimed $1.0 \mathrm{~mm}$ above the gustatory neocortex [anteroposterior, $+1.2 \mathrm{~mm}$ relative to bregma; lateral, $\pm 5.5 \mathrm{~mm}$; ventral, $5.5 \mathrm{~mm}$ (Paxinos and Watson, 1986)]. The cannulas were positioned in place with acrylic dental cement and secured by two skull screws. A stylus was placed in the guide cannulas to prevent clogging. Animals were allowed 1 week to recuperate before being subjected to experimental manipulations. The stylus was removed from the guide cannulas, and a 28 gauge injection cannula, extending $1.0 \mathrm{~mm}$ from the tip of the guide cannula, was inserted. The injection cannula was connected via PE20 tubing to a Hamilton microsyringe driven by a microinfusion pump (CMA/100; Carnegie Medicin). Microinjection was performed bilaterally in a $1 \mu$ l volume per hemisphere delivered over 1 min. The injection cannula was left in position before withdrawal for an additional $1 \mathrm{~min}$ to minimize dragging of the injected liquid along the injection tract.
Homogenization. Rats were decapitated at the indicated times after the completion of the exposure to the unfamiliar taste. The insular cortex containing the gustatory cortex, or other brain areas indicated in Results, were dissected out. For the insular cortex, the crossing of the rhinal fissure and the medial cerebral artery was used as a reference point, and cortical tissue $1.5 \mathrm{~mm}$ deep, $1.5 \mathrm{~mm}$ rostral, $1.0 \mathrm{~mm}$ caudal, and $2.0 \mathrm{~mm}$ dorsal to it was excised. The tissue was immediately homogenized in a glass Teflon homogenizer in $200 \mu \mathrm{l}$ of SDS sample buffer (10\% glycerol, $5 \% \beta$-mercaptoethanol, and $2.3 \%$ SDS, in $62.5 \mathrm{~mm}$ Tris-HCl, $\mathrm{pH} 6.8$ ) and boiled for $5 \mathrm{~min}$. Samples were immediately stored at $-20^{\circ} \mathrm{C}$ until further usage.

Western blot analysis. Aliquots in SDS sample buffer were subjected to SDS-PAGE (8\% polyacrylamide) (Laemmli, 1970) and Western blot analysis (Burnette, 1981). The amount of protein in each sample was always determined before loading, and the same amount of protein $(40-60 \mu \mathrm{g})$ was loaded in each lane (Lowry et al., 1951). After the run, the lanes were also compared by Ponceau staining. After it was blocked with $1 \% \mathrm{BSA}$ for $1 \mathrm{hr}$ at room temperature, the blot was reacted overnight at $4^{\circ} \mathrm{C}$ or for $2 \mathrm{hr}$ at room temperature with the primary antibody. The blots were subsequently incubated for $1 \mathrm{hr}$ at room temperature with HRP-linked protein A or with goat anti-mouse HRPlinked antibody before exposure to the ECL substrate. The antibodies directed against the phosphorylated proteins were usually applied first (dpERK, 1:30,000; pElk-1, 1:1000; dpJNK, 1:1000; pAkt, 1:1000; dpp38MAPK, 1:1000). The blots were then stripped in $0.9 \% \mathrm{w} / \mathrm{v} \mathrm{NaCl}, 100$ $\mathrm{mm}$ Tris- $\mathrm{HCl}, 1 \% \mathrm{v} / \mathrm{v}$ Tween-20, and $2 \% \mathrm{w} / \mathrm{v}$ SDS, $\mathrm{pH} 7.6$, four times for $15 \mathrm{~min}$ each at room temperature under vigorous shaking. The blots were then rinsed three times for $10 \mathrm{~min}$ in washing buffer (same stripping buffer with $0.05 \%$ Tween-20 instead of SDS), blocked for $1.5 \mathrm{hr}$ with $1 \%$ $\mathrm{BSA}$, and incubated with the antibodies corresponding to the phosphorylation state-independent forms of the proteins as described above (ERK, 1:2000; Elk-1, 1:1000; JNK, 1:1000; Akt, 1:1000; p38MAPK, $1: 1000)$. The efficacy of the stripping step was assessed by omitting the first antibody and verifying the lack of signals on the blot. Quantification was performed using a computerized densitometer and image analyzer (Molecular Dynamics, Sunnyvale, CA).

Statistics. Differences among the groups were determined using oneway ANOVA. For paired comparisons, Scheffe contrast tests were used with an $\alpha$ level of 0.05 .

\section{RESULTS}

\section{Drinking an unfamiliar taste activated ERK1-2 in the insular cortex}

Samples of the insular cortex from rats that were allowed to drink a solution of unfamiliar taste, i.e., $10 \mathrm{ml}$ of $0.1 \%$ saccharin, were immunoblotted with a specific dpERK1-2 antibody. ERK activation was detected at 10 min after the offset of drinking saccharin, peaking at $30 \mathrm{~min}$, and subsiding to basal levels within $60 \mathrm{~min}$ (Fig. 1A). The total amount of ERK1-2 was not affected by drinking the novel taste solution (Fig. $1 B$ ). The effect on dpERK1-2 was not restricted to saccharin; a similar increase in ERK activation was observed in the insular cortex after sampling other unfamiliar tastes: a solution of $1 \% \mathrm{NaCl}$ [dpERK $(\mathrm{NaCl}) /$ $\operatorname{dpERK}($ water $)=2.0 \pm 0.3 ; n=6]$ or a solution of $1 \%$ glycine $[\mathrm{dpERK}$ (glycine)/dpERK (water) $=2.4 \pm 0.5 ; n=6]$. ERK activation was not detected in the occipital lobe, olfactory bulb, piriform cortex, or cerebellum (Fig. 2).

\section{A familiar taste had no effect on ERK activation}

A single exposure to an unfamiliar taste significantly attenuates the effectiveness of subsequent CTA training with that same taste (Rosenblum et al., 1993), as expected from latent inhibition (Lubow, 1989). Such preexposure also essentially abolished the effect of drinking the saccharin solution on the activation of ERK1-2 in the insular cortex (Fig. $1 A$, inset). The activation of ERK1-2 as a function of time was similar when plotted either as dpERK (saccharin, unfamiliar)/dpERK (saccharin, familiar) or as dpERK (saccharin, unfamiliar)/dpERK (water) (Fig. 1A). Again, the total amount of ERK1-2 was unaffected (Fig. 1B). 


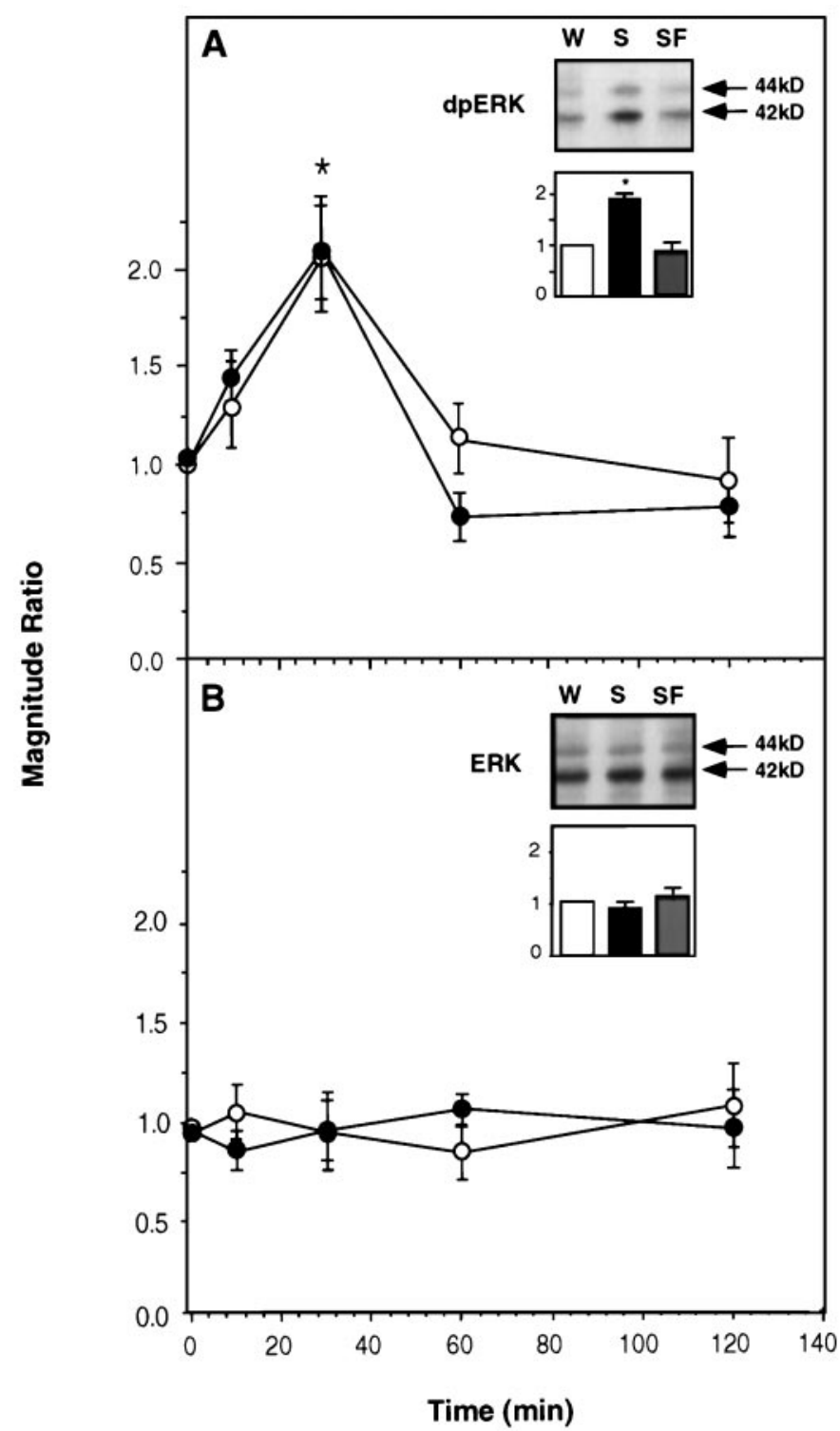

Figure 1. The effect of gustatory experience on activation of ERK1-2 in the rat insular cortex. $A$, Activated ERK1-2 (dpERK1-2). B, Total ERK1-2. Three types of experimental groups were used. In group 1, rats were allowed to drink water. In group 2, rats were allowed to drink a solution of saccharin, a taste that they had never encountered before (the unfamiliar taste). In group 3, rats were allowed to drink the saccharin solution, which in this case was made familiar earlier by allowing them to drink it once for $10 \mathrm{~min} 3 \mathrm{~d}$ before presenting it again in the experiment (see Materials and Methods). Several sets of experiments were conducted in different combinations of the groups. Each graph depicts a "magnitude ratio," which is the ratio of the combined intensity of the $42+44 \mathrm{kDa}$ bands in one experimental group over another $\left(\right.$ group $\left._{\mathrm{i}} / \mathrm{group}_{\mathrm{j}}\right)$ as a function of time after the offset of drinking. A, Filled circles, Data from a set of experiments depicting group $2 /$ group $_{1}$, i.e., the ratio of dpERK1-2 in the insular cortex of rats allowed to drink unfamiliar saccharin solution over that in rats allowed to drink water. A, Open circles, Data from another set of experiments depicting group $_{2} /$ group $_{3}$, i.e., the ratio of dpERK1-2 in the insular cortex of rats allowed to drink unfamiliar saccharin over that in rats allowed to drink familiar saccharin. Inset, A representative blot of dpERK1-2 at $t=30$ min after drinking water $(W)$, unfamiliar saccharin $(S)$, or familiar saccharin $(S F)$, and quantification of experiments similar to those depicted in the blot (activation in the water group, $1.0 ; n=9-11$ each). The results show that the effect of taste experience on the activation of ERK1-2 in the insular cortex is attributable to the unfamiliarity of the taste. $B$, Same as in $A$ but depicting the results for total ERK1-2. (In this and all other figures, values are mean \pm SEM, and ${ }^{*} p<0.05$.)
A
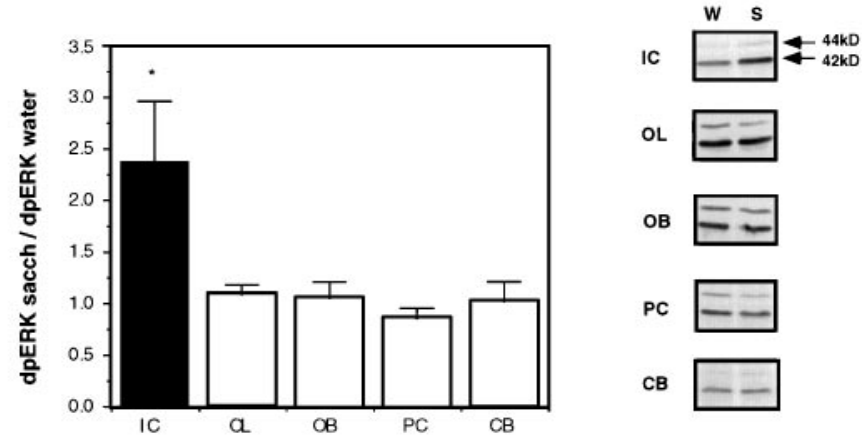

B

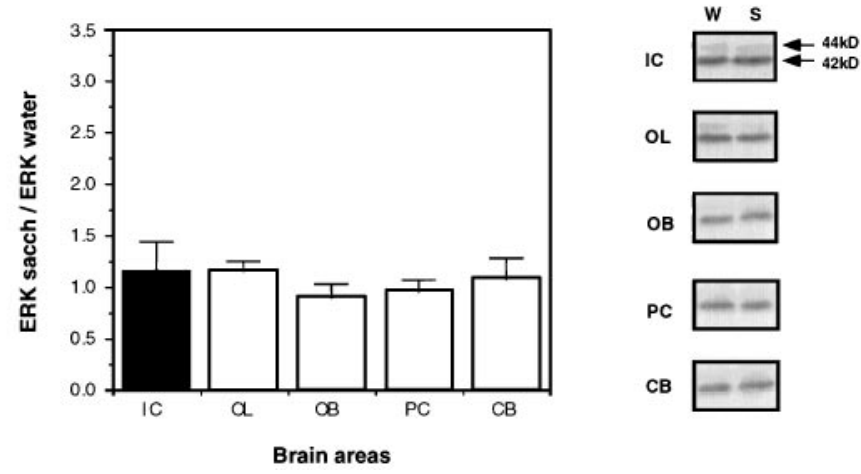

Figure 2. The level of activated ERK1-2 and total ERK1-2 in various brain regions $30 \mathrm{~min}$ after the exposure to the unfamiliar taste. $A$, Activated ERK1-2. B, Total ERK1-2. IC, Insular cortex; $O L$, occipital lobe; $O B$, olfactory bulb; $P C$, piriform cortex; $C B$, cerebellum. $n=6$ in each group.

\section{An MEK inhibitor impaired CTA memory in the insular cortex}

To determine whether the activation of ERKs was not only correlated but also obligatory for taste learning, we locally microinjected an inhibitor of MEK (MAPK/ERK kinase), PD098059 (Alessi et al., 1995; Dudley et al., 1995), into the insular cortex 30 min before exposure to the novel taste in the context of CTA training. As seen in Figure 3, the MEK inhibitor impaired longterm CTA memory tested 72 or $120 \mathrm{hr}$ after training, without significantly affecting memory at $3 \mathrm{hr}$ after training (short-term memory) (Lamprecht and Dudai, 1997). The MEK inhibitor also attenuated the effect of preexposure to the novel taste in an incidental learning situation, assayed in the latent inhibition paradigm (see Materials and Methods). Thus, the AI of rats microinjected with PD098059 into the insular cortex $30 \mathrm{~min}$ before preexposure to saccharin $3 \mathrm{~d}$ prior to CTA training, and tested $3 \mathrm{~d}$ after training, was $90 \pm 4$ versus $75 \pm 5$ in controls microinjected into the insular cortex with ACSF $(p<0.05)$ and $97 \pm 4$ in untreated controls subjected to CTA training in the absence of a latent inhibition training $(n=6-7$ each). The inhibitor had no effect on saccharin preference in noncontingent controls: the AI of rats microinjected into the insular cortex with PD098059 30 min before exposure to saccharin in the absence of subsequent $\mathrm{LiCl}$ intraperitoneal injection, and tested for saccharin versus water preference $72 \mathrm{hr}$ later, was $43 \pm 5$ compared with $40 \pm 5$ in rats subjected to the same protocol but microinjected into the insular cortex with ACSF instead of PD098059 and $44 \pm 4$ in naive untreated rats ( $n=6$ per group). These data, combined with the data of Figure 3, also indicate that the MEK inhibitor blocks $\sim 40 \%$ of CTA memory at 72 or $120 \mathrm{hr}$, an effect compa- 


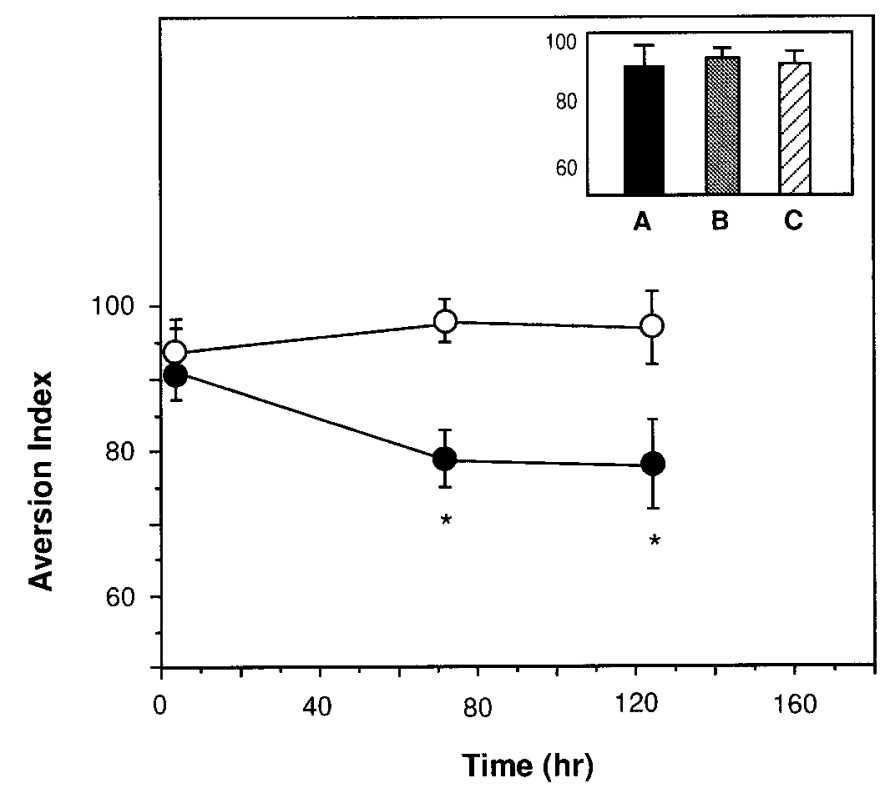

Figure 3. Impairment of CTA by local microinjection of the reversible MEK inhibitor PD098059 into the insular cortex. AI values are plotted versus time after training. Each group of animals was tested only once at the indicated time. Filled circles, Rats microinjected with $1 \mu \mathrm{l}$ of $30 \mu \mathrm{M}$ PD089059 per hemisphere into the insular cortex 30 min before CTA training; open circles, rats microinjected with $1 \mu \mathrm{l}$ of ACSF plus $1 \%$ DMSO $(n=6$ each). Inset, Spatial and temporal specificity of the inhibitor effect. $A$, Control animals. $B$, PD098059 microinjected $2 \mathrm{~mm}$ above the stereotaxic coordinates used for injection into the insular cortex. $C$, PD098059 microinjected into the insular cortex $30 \mathrm{~min}$ before the first memory test. $n=8$ in each group.

rable in magnitude to that of inhibitors of protein synthesis in the insular cortex (Rosenblum et al., 1993).

The MEK inhibitor also did not impair retrieval of long-term CTA memory once formed, implying no effect on sensory, motivational, or motor abilities required for CTA expression (Fig. 3, inset). The effect on CTA was not observed when the inhibitor was injected $2 \mathrm{~mm}$ above the insular cortex (Fig. 3, inset). When PD098059-microinjected animals were retrained in a CTA paradigm (this time using $1 \%$ glycine as the novel taste) 1 week after the completion of the first CTA experiment, they displayed normal CTA (AI, $96 \pm 4$ vs $97 \pm 5$, control vs PD098059-injected animals), indicating that the effect of the inhibitor was transient and reversible and that the insular cortex suffered no long-term damage.

To confirm that PD098059 was indeed capable of blocking taste-induced ERK activation in the insular cortex in vivo, we microinjected the inhibitor $30 \mathrm{~min}$ before the exposure of the animals to the novel taste, removed the insular cortex $30 \mathrm{~min}$ later, and immunoblotted its homogenate as described above. We found that the inhibitor blocked the taste-induced increase in ERK activity in vivo (Fig. 4).

\section{Elk-1, an ERK downstream substrate, was also activated by taste experience}

Elk-1 is a member of the ternary complex factor, which together with the serum response factor (SRF) forms a multiprotein complex at the serum response element (SRE) of the c-fos promoter (Hipskind et al., 1991; Treisman, 1995). Elk-1 is rapidly phosphorylated in response to activation of ERKs, JNKs, and p38MAPK (Whitmarsh et al., 1995, 1997). Phosphorylation of
A

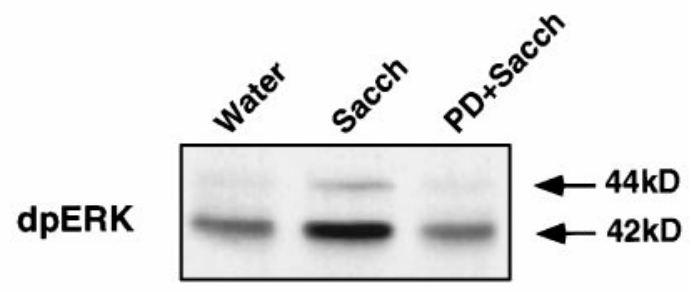

B

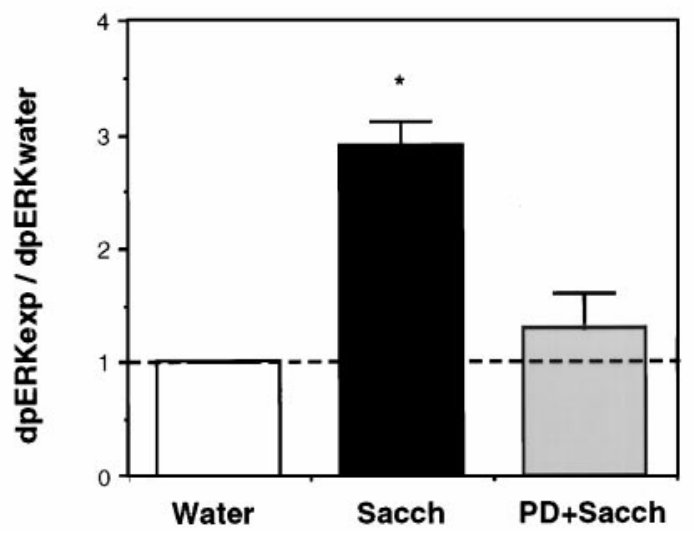

Figure 4. Inhibition of taste-dependent ERK1-2 activation by PD098059 in the insular cortex. $A$, A representative blot of samples of insular cortex of animals subjected to different manipulations. Water, Animals drinking $10 \mathrm{ml}$ of water; Sacch, animals exposed to $10 \mathrm{ml}$ of saccharin; $P D+$ Sacch, animals injected with PD098059 $30 \mathrm{~min}$ before the exposure to saccharin. Rats were killed 30 min after each treatment, and the samples were blotted with anti-diphospho ERK1-2 antibodies. $B$, Summary of data from experiments like those in $A$. Open bar, Animals exposed to water; filled bar, animals exposed to saccharin; shaded bar, animals microinjected into the insular cortex with PD098059 and 30 min later presented with saccharin. $n=4$ per group.

Elk-1 increases its ability to form a ternary complex with SRE and SRF and ultimately to activate c-fos transcription (Janknecht et al., 1994; Gille et al., 1995). We therefore decided to check whether this member of the transcription factor family undergoes activation in the rat insular cortex after sampling a novel taste. By using a specific anti-phospho Elk-1 (Ser383) antibody, we found a taste-induced increase in the level of phosphorylation of Elk-1 (Fig. 5), which correlated temporally with the increase of ERK activity. Activation of Elk-1 was abolished by familiarity with the taste (Fig. 5) and was not detected in the cerebellum, occipital lobe, olfactory bulb, and piriform cortex (data not shown).

\section{Taste experience also activated JNK1-2 but not Akt kinase or p38MAPK}

JNK (also known as SAPK) was also shown to activate Elk-1 and increase c-Fos induction after exposure of cells to different agents, such as UV radiation, cytokines, and growth factors (Gille et al., 1995; Whitmarsh et al., 1995; Su and Karin, 1996; Yang et al., 1998). By using phospho-specific anti-JNK antibodies, we observed activation of JNK1-2 60 min after drinking the taste solution, subsiding to basal level within an additional $60 \mathrm{~min}$ (Fig. 6). The total amount of JNK remained unaltered (Fig. 6).

In contrast, Akt, a serine-threonine kinase (PKB) activated by the phosphoinositide 3-kinase (PI3-K) cascade (Marte and Downward, 1997; Downward, 1998), and p38MAPK, the vertebrate HOG homolog (Su and Karin, 1996), were not activated in 


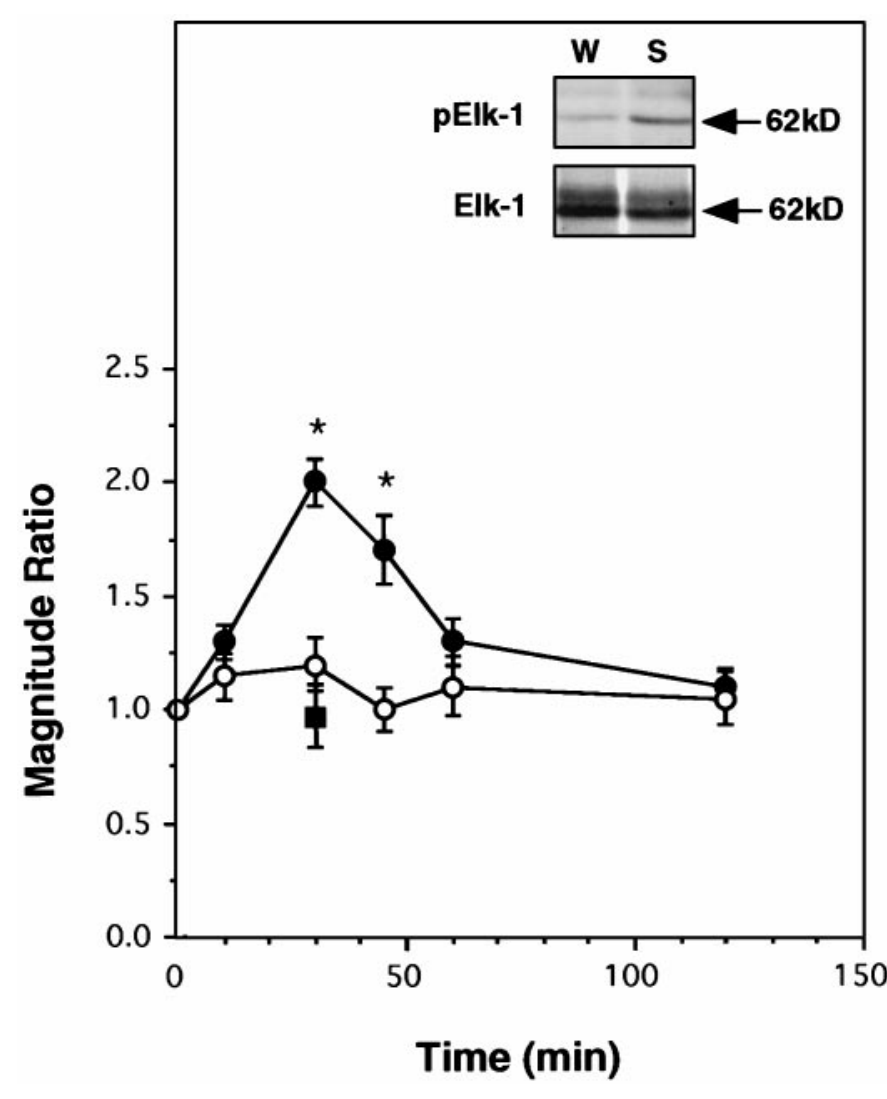

Figure 5. Activation of Elk-1 in the insular cortex as a function of time after sampling a saccharin solution as an unfamiliar taste. Magnitude ratio is expressed as pElk-1 (saccharin)/pElk-1 (water) (filled circles), Elk-1 (saccharin)/Elk-1 (water) (open circles), or pElk-1 (saccharin)/pElk-1 (familiar saccharin) (filled square, $t=30 \mathrm{~min}$ ). In the latter case, the saccharin solution was made familiar by preexposure, as detailed in the legend to Figure 1. $n=8-12$ per time point.

the insular cortex after drinking the unfamiliar taste (Fig. 7). The total amount of these two signaling kinases was not altered as well (Fig. 7).

\section{DISCUSSION}

We have approached the role of MAPKs in taste learning in the insular cortex of the behaving rat by using two methodologies. One was correlation of MAPK activation, quantified by immunoblotting, with a behavioral experience. The other was inference of function from dysf unction, using local microinjections into the insular cortex of a reversible MAPK-kinase inhibitor. We found that the ERK signaling cascade in the insular cortex is necessary for taste learning and that the ERK, as well as the JNK cascade, is activated in the insular cortex by a taste experience that results in long-term taste memory. In contrast, other MAPKs, Akt/ $\mathrm{PKB}$, and $\mathrm{p} 38 \mathrm{MAPK}$, were unaffected. Together, the data show that MAPK cascades are activated specifically and differentially in taste learning in the insular cortex and that at least part of this activation is obligatory for long-term, but not short-term, taste memory. Several laboratories have provided evidence that the insular cortex plays a role in taste learning and memory (Braun et al., 1972; Dunn and Everitt, 1988; Bermudez-Rattoni and McGaugh 1991, Gallo et al., 1992; Kiefer and Orr, 1992; Rosenblum et al., 1993, 1997; Schafe and Bernstein, 1998). The fine delineation of the insular cortex subregion(s) required for CTA learning, and a robust correspondence with neuronal layers directly impli-

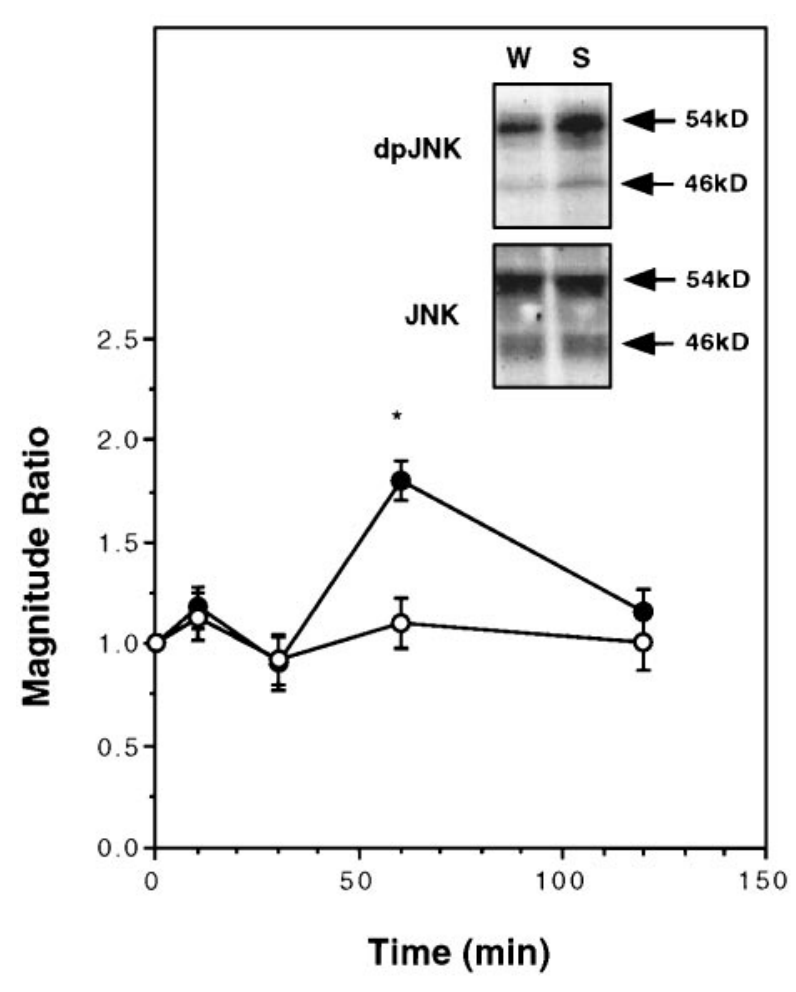

Figure 6. Activation of JNK1-2 in the insular cortex as a function of time after sampling a novel taste. Magnitude ratio is expressed as dpJNK (saccharin)/dpJNK (water) or JNK (saccharin)/JNK (water), respectively (combined values for both bands in each case). Inset, A representative blot at $t=60$ min after drinking. Filled circles, Diphospho-JNK; open circles, total JNK. $n=9-12$ per time point.

cated in the processing of taste attributes by electrophysiological analysis, must yet be clearly established (Lasiter and Glanzman 1982; Neard et al., 1996). The role of the insular cortex in CTA is especially important in situations in which the conditioned taste is unfamiliar (Yamamoto et al., 1994; Rosenblum et al., 1997). Anatomical, cytotoxic, or metabolic lesions of insular cortex inflicted during the acquisition of taste information in CTA training usually do not abolish subsequent CTA memory, but rather attenuate it considerably (Gallo et al., 1992; Rosenblum et al., 1993, 1997; Yamamoto et al., 1994). This is consistent with our data showing that inhibition of MEK activity in the insular cortex during the taste acquisition phase of CTA training results in a marked reduction, but not elimination, of long-term CTA memory (see Results). The present findings thus corroborate the notion that the insular cortex participates in the processing and storage of information about new taste experiences and identify molecular cascades that take part in the aforementioned processes.

Investigations of MAPKs in the nervous system often focus on cell cultures or relatively simple preparations (for review, see Seger and Krebs, 1995; Su and Karin, 1996). In cultured mammalian neurons, the activation of ERKs is regulated by membrane depolarization and calcium influx (Rosen et al., 1994), G-protein-coupled receptors (Sugden and Clerk, 1997), and cytoskeletal reorganization (Irigoyen et al., 1997). It is also regulated by muscarinic agonists in oligodendrocyte progenitors (Larocca and Almazan, 1997). ERK pathways were implicated in long-term facilitation and in apCAM learning-related internalization in Aplysia sensory neurons (Bailey et al., 1997; Martin et al., 
A

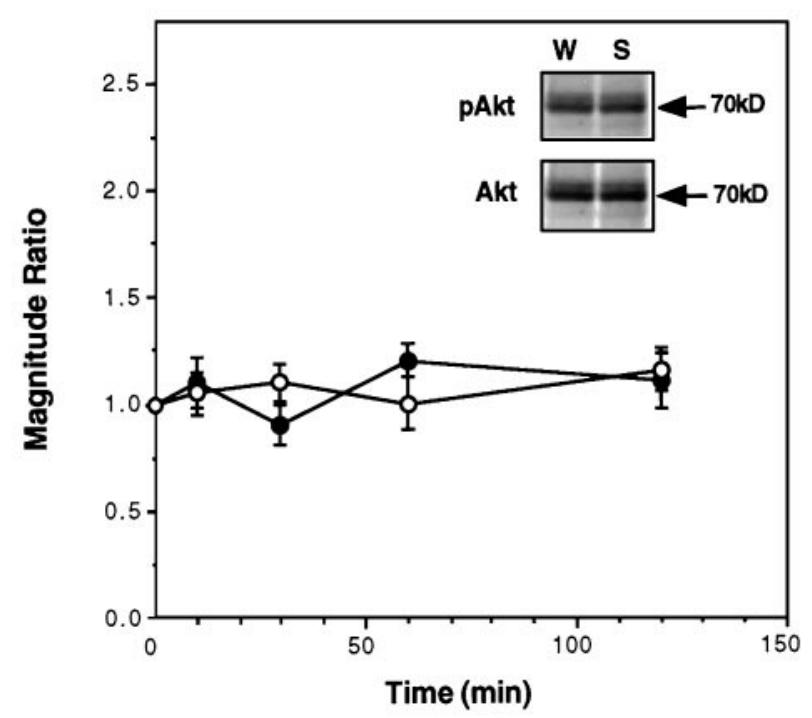

B

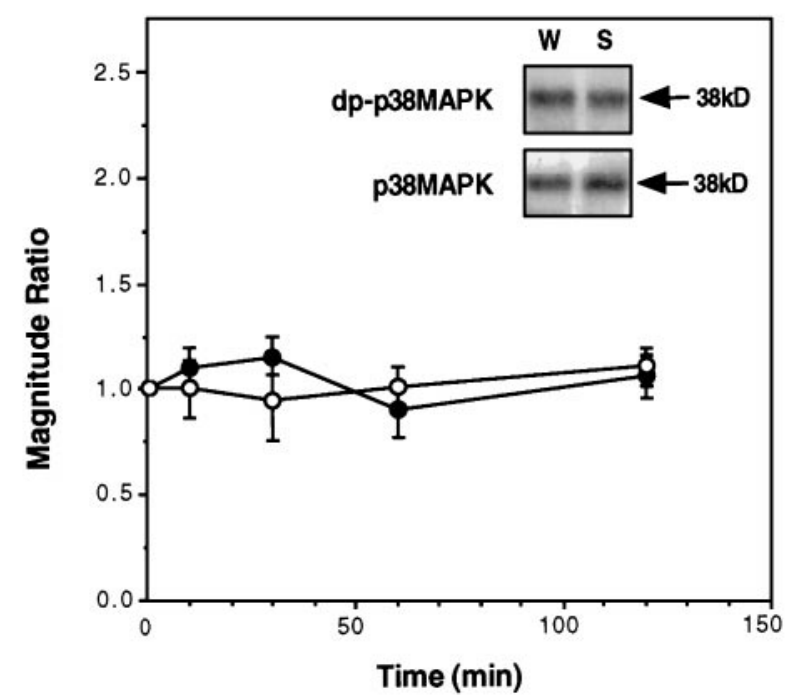

Figure 7. The effect of gustatory experience on Akt and p38MAPK in the insular cortex. $A$, The level of phospho-Akt ( $p A k t)$ and total Akt as a function of time after drinking saccharin or water. Filled circles, pAkt; open circles, total Akt. Inset, A representative blot at $t=30$ min after drinking. $B$, The level of diphospho-p38MAPK ( $d p-p 38 M A P K)$ and total p38MAPK as a function of time after drinking saccharin or water. Filled circles, dp-p38MAPK; open circles, total p38MAPK. Inset, A representative blot at $t=30$ min after drinking. In both $A$ and $B, n=11-15$ per time point.

1997; Michael et al., 1998), and in classical conditioning in Hermissenda (Crow et al., 1998). Activation of p42MAPK was reported to take place in brain slices in area CA1 during hippocampal LTP, a candidate plasticity mechanism in the mammalian brain (English and Sweatt, 1996). However, to the best of our knowledge, no studies were reported on the role of the MAPK signaling cascades in learning in vertebrates. Here, we show that activation of MAPK signaling cascades is correlated with and critical for long-term storage of experience-dependent information in the cortex of the behaving rat in natural learning situations.

That the taste input did not induce massive, nondiscriminative activation of MAPKs signaling cascades in cortex is evident from the observation that the stress-related p38MAPK pathway, as well as the Akt pathway involving the PI3 kinase cascade, were not affected. The activation of the ERK pathway might involve nuclear substrates [although an effect on a cytoplasmic substrate cannot be excluded (Bailey et al., 1997)]. Indeed, we found that a nuclear target of ERKs, the transcription factor Elk-1, underwent activation during exposure of rats to a novel taste, and this activation was temporally correlated with that of ERK. Activation of Elk-1 was recently shown to result from electrical stimulation of the glutamatergic corticostriatal pathway in the rat brain (Sgambato et al., 1998). ERKs, as well as JNKs and p38MAPK, are differentially targeted to the ETS-domain of Elk-1, resulting in a specific activation of the transcription factor, depending on the stimulating input (Yang et al., 1998). Because we did not observe a temporal correlation between Elk-1 and JNK activation, nor any correlation with p38MAPK, we conclude that in the insular cortex, taste-dependent Elk-1 activation depends on ERKs. As to JNKs, their taste-dependent activation in the insular cortex might be related to cytoskeletal reorganization (Irigoyen et al., 1997), potentially reflecting some cellular remodeling processes.

All in all, our data indicate that novel, potentially salient taste information activates selected sets of intracellular signaling cascades in the insular cortex. Combinatorial stimulation of MAPKs cascades might lead to a differential activation of transcription factors, resulting in specific modulation of gene transcription. For example, the interplay between ERKs and JNKs activation could result in a differential expression of the c-Fos and c-Jun transcription factors, members of the AP-1 transcriptional complex (Karin, 1996). Specifically, we propose that activation of the ERK signaling cascade is related to the encoding of novelty and/or saliency during acquisition and to the initiation of long-term storage of some aspects of taste memory in the insular cortex. The contextual encoding of novelty and saliency in the insular cortex during the encounter with the unfamiliar taste, and the encoding of information about it, is expected to involve neuromodulatory systems, e.g., the cholinergic basal forebrain input (Naor and Dudai, 1996). A plausible model is that activation of the cholinergic basal forebrain system, and the subsequent release of acetylcholine in the cortex, leads to activation of muscarinic receptors that trigger ERK activation via PKC (Bahar and Dudai, 1997; Yasoshima and Yamamoto, 1997) or PYK2 (Lev et al., 1995). Calcium influx via NMDA receptors, which are necessary for the encoding (but not the retrieval) of taste memory in the insular cortex (Rosenblum et al., 1997), might also act synergistically with the cholinergic-dependent signal transduction cascades to activate ERKs.

The present findings could be construed as being in line with the hypothesis that modulation of gene expression, protein synthesis, and growth-related processes are involved in long-term experience-dependent plasticity in the nervous system (Goelet et al., 1986; Bailey and Kandel, 1993). However, we do not know yet whether the changes that we detect in MAPKs activation in the insular cortex indeed culminate in modulation of gene expression and growth processes that are causally related to the encoding of memory in that brain region. Furthermore, substantial support for an obligatory role of gene expression in the formation of longterm memory stems from the observation that antibiotics that inhibit protein synthesis also inhibit memory consolidation (Davis and Squire, 1984; Montarolo et al., 1986). However, antibiotics, such as anisomycin, which is extensively used as a consol- 
idation blocker, are also potent activators of MAPKs (Kyriakis et al., 1994). It is still to be determined whether the effects of antibiotics on long-term memory in vivo is attributable to their interaction with MAPKs, with the translational apparatus, or both.

\section{REFERENCES}

Alessi DR, Cuenda A, Cohen P, Dudley DT, Saltiel AR (1995) PD 098059 is a specific inhibitor of the activation of mitogen-activated protein kinase kinase in vitro and in vivo. $\mathrm{J}$ Biol Chem 270:27489-27494.

Bahar A, Dudai Y (1997) Molecular mechanisms of conditioned taste aversion memory in the rat insular cortex: potential involvement of protein kinase C. Soc Neurosci Abstr 23:823.

Bailey CH, Kandel ER (1993) Structural changes accompanying memory storage. Annu Rev Physiol 55:397-426.

Bailey CH, Kaang BK, Chen M, Martin KC, Lim CS, Casadio A, Kandel ER (1997) Mutation in the phosphorylation sites of MAP kinase blocks learning-related internalization of apCAM in Aplysia sensory neurons. Neuron 18:913-924.

Bermudez-Rattoni F, McGaugh JL (1991) Insular cortex and amygdala lesions differentially affect acquisition on inhibitory avoidance and conditioned taste aversion. Brain Res 594:165-170.

Braun JJ, Slick TB, Lorden JF (1972) Involvement of gustatory neocortex in the learning of taste aversions. Physiol Behav 9:637-641.

Braun JJ, Lasiter PS, Kiefer SW (1982) The gustatory neocortex of the rat. Physiol Psychol 10:13-54.

Bures J, Buresova O, Krivanek J (1988) Brain and behavior: paradigms for research in neural mechanisms. New York: Wiley.

Burnette WW (1981) Western blotting: electrophoretic transfer of proteins from sodium dodecyl sulfate-polyacrilamide gels to unmodified nitrocellulose and radiographic detection with antibody and radioiodinated protein A. Anal Biochem 112:195-203.

Cano E, Mahadevan L (1995) Parallel signal processing among mammalian MAPKs. Trends Biochem Sci 20:117-122.

Crow T, Xue-bian JJ, Siddiqi V, Kang Y, Neary JT (1998) Phosphorylation of mitogen-activated protein kinase by one-trial and multi-trial classical conditioning. J Neurosci 18:3480-3487.

Davis HP, Squire LR (1984) Protein synthesis and memory: a review. Psychol Bull 96:518-559.

Downward J (1998) Lipid-regulated kinases: some common themes at last. Science 279:673-674.

Dudley DT, Pang L, Decker SJ, Bridges AJ, Saltiel AR (1995) A synthetic inhibitor of the mitogen-activated protein kinase cascade. Proc Natl Acad Sci USA 92:7686-7689.

Dunn LT, Everitt BJ (1988) Double dissociation of the effects of amygdala and insular cortex lesions on conditioned taste aversion, passive avoidance, and neophobia in the rat using the excitotoxin ibotenic acid. Behav Neurosci 102:3-23.

English JD, Sweatt JD (1996) Activation of p42 mitogen-activated protein kinase in hippocampal long term potentiation. J Biol Chem 271:24329-24332.

Finger TE (1987) Gustatory nuclei and pathways in the central nervous system. In: Neurobiology of taste and smell (Finger TE, Silver WL, eds), pp 331-353. New York: Wiley.

Gallo M, Roldan G, Bures J (1992) Differential involvement of gustatory insular cortex and amygdala in the acquisition and retrieval of conditioned taste aversion in rats. Behav Brain Res 52:91-97.

Garcia J, Kimmerldorf DJ, Koelling RA (1955) Conditioned aversion to saccharin resulting from exposure to gamma radiation. Science 122:157-158.

Gille H, Kortenjann M, Thomao O, Moomaw C, Slaughter C, Cobb MH, Shaw PE (1995) ERK phosphorylation potentiates Elk-1 mediated ternary complex factor formation and transactivation. EMBO J 14:951-962.

Goelet P, Castellucci VF, Schacher S, Kandel ER (1986) The long and the short of long-term memory - a molecular framework. Nature 322:419-422.

Hebb DO (1949) The organization of behavior: a neuropsychological theory. New York: Wiley.

Hettinger TP, Frank ME (1992) Information processing in mammalian gustatory cortex. Curr Opin Neurobiol 2:469-478.

Hipskind RA, Rao VN, Mueller CGF, Reddy ESP, Nordheim A (1991) Ets-related protein Elk-1 is homologous to the c-fos regulatory factor p62 ${ }^{\mathrm{TCF}}$. Nature 354:531-534.
Irigoyen JP, Besser D, Nagamine Y (1997) Cytoskeletal reorganization induces the urokinase-type plasminogen activator gene via the ras/ extracellular signal-regulated kinase signaling pathway. J Biol Chem 272:1904-1909.

Janknecht R, Zinck R, Ernst WH, Nordheim A (1994) Functional distinction of the transcription factor Elk-1. Oncogene 9:1273-1278.

Karin M (1996) The regulation of AP-1 activity by mitogen-activated protein kinases. Phil Trans R Soc Lond B Biol Sci 351:127-134.

Kiefer SW, Orr MR (1992) Taste avoidance, but not aversion, learning in rats lacking gustatory cortex. Behav Neurosci 106:140-146.

Kyriakis JM, Banerjee P, Nikolakaki E, Dai T, Rubie EA, Ahmad MF, Avruch J, Woodgett JR (1994) The stress-activated protein kinase subfamily of c-jun kinases. Nature 369:156-160.

Laemmli UK (1970) Cleavage of structural proteins during the assembly of the head of bacteriophage $\mathrm{T}_{4}$. Nature 227:680-685.

Lamprecht R, Dudai Y (1997) cAMP response element-binding protein in the amygdala is required for long- but not short-term conditioned taste aversion memory. J Neurosci 17:8443-8450.

Larocca JN, Almazan G (1997) Acetylcholine agonists stimulate mitogen-activated protein in kinase in oligodendrocyte progenitors by muscarinic receptors. J Neurosci Res 50:743-754.

Lasiter PS, Glanzman DL (1982) Cortical substrates of taste aversion learning: dorsal prepiriform (insular) lesions disrupt taste aversion learning. J Comp Physiol Psychol 96:376-392.

Lev S, Moreno H, Martinez R, Canoll P, Peles E, Musacchio JM, Plowman GD, Rudy B, Schlessinger J (1995) Protein tyrosine kinase PYK2 involved in $\mathrm{Ca}^{++}$-induced regulation of ion channel and MAP kinase functions. Nature 376:737-745.

Lowry OH, Rosebrough NJ, Farr AL, Randall RJ (1951) Protein measurement with the Folin phenol reagent. J Biol Chem 193:265-275.

Lubow RE (1989) Latent inhibition and conditioned attention theory. London: Cambridge UP.

Marte BM, Downward J (1997) PKB/Akt: connecting phosphoinositide 3-kinase to cell survival and beyond. Trends Biochem Sci 22:355-358.

Martin KC, Michael D, Rose JC, Barad M, Casadio A, Zhu H, Kandel ER (1997) MAPK kinase translocates into the nucleus of the presynaptic cell and is required for long-term facilitation in Aplysia. Neuron 18:899-912.

Michael D, Martin KC, Seger R, Ning MN, Baston R, Kandel ER (1998) Repeated pulses of serotonin required for long-term facilitation activate mitogen-activated protein kinase in sensory neurons of Aplysia. Proc Natl Acad Sci USA 95:1864-1869.

Montarolo PG, Goelet P, Castellucci VF, Morgan J, Kandel ER, Schacher S (1986) A critical period for macromolecular synthesis in long-term heterosynaptic facilitation in Aplysia. Science 234:1239-1254.

Naor C, Dudai Y (1996) Transient impairment of cholinergic function in the rat insular cortex disrupts the encoding of taste in conditioned taste aversion. Behav Brain Res 79:61-67.

Neard L, Ramirez-Amaya V, Ormsby CE, Bermudez-Rattoni F (1996) Differential effects of anterior and posterior insular cortex lesions on the acquisition of conditioned taste aversion and spatial learning. Neurobiol Learn Mem 66:44-50.

Paxinos G, Watson C (1986) The rat brain in stereotaxic coordinates, Ed 2. New York: Academic.

Revusky S, Garcia J (1970) Learned associations over long delays. Psychol Learn Motiv 4:3-84.

Rosen L, Ginty DD, Weber MJ, Geenberg ME (1994) Membrane depolarization and calcium influx stimulate MEK and MAP kinase via activation of ras. Neuron 12:1207-1221.

Rosenblum K, Meiri N, Dudai Y (1993) Taste memory: the role of protein synthesis in gustatory cortex. Behav Neural Biol 59:49-56.

Rosenblum K, Berman DE, Hazvi S, Lamprecht R, Dudai Y (1997) NMDA receptor and the tyrosine phosphorylation of its $2 \mathrm{~B}$ subunit in taste learning in the rat insular cortex. J Neurosci 17:5129-5135.

Schafe GE, Bernstein IL (1998) Forebrain contribution to the induction of a brainstem correlate of conditioned taste aversion. II. Insular (gustatory) cortex. Brain Res 800:40-47.

Schafe GE, Seeley R, Bernstein IL (1995) Forebrain contribution to the induction of a cellular correlate of conditioned taste aversion in the nucleus of the solitary tract. J Neurosci 15:6791-6796.

Seger R, Krebs EG (1995) The MAPK signaling cascade. FASEB J 9:726-735.

Sgambato V, Vanhoutte P, Pages C, Rogard M, Hipskind R, Besson MJ, Caboche J (1998) In vivo expression and regulation of Elk-1, a target 
of the extracellular-regulated kinase signaling pathway, in the adult rat brain. J Neurosci 18:214-226.

Su B, Karin M (1996) Mitogen-activated protein kinase cascades and regulation of gene expression. Curr Opin Immunol 8:402-411.

Sugden PH, Clerk A (1997) Regulation of the ERK subgroup of MAPK kinase cascades through G protein-coupled receptors. Cell Signalling 9:337-351.

Treisman R (1995) Journey to the surface of the cell: Fos regulation and the SRE. EMBO J 14:4905-4913.

Whitmarsh AJ, Shore P, Sharrocks AD, Davis RJ (1995) Integration of MAP kinase signal transduction pathways at the serum response element. Science 269:403-407.
Whitmarsh AJ, Yang SH, Su MSS, Sharrocks AD, Davis RJ (1997) Role of p38 and JNK MAP kinases in the activation of ternary complex factors. Mol Cell Biol 17:2360-2371.

Yamamoto T, Shimura T, Sako N, Yasoshima Y, Sakai N (1994) Neural substrates for conditioned taste aversion in the rat. Behav Brain Res 65:123-137.

Yang SH, Whitmarsh AJ, Davis RJ, Sharrocks AD (1998) Differential targeting of MAP kinases to the ETS-domain transcription factor Elk-1. EMBO J 17:1740-1749.

Yasoshima Y, Yamamoto T (1997) Rat gustatory memory requires protein kinase $\mathrm{C}$ activity in the amygdala and cortical gustatory area. NeuroReport 8:1363-1367. 